\title{
BMJ A randomised trial comparing the cost Open effectiveness of different emergency department healthcare professionals in soft tissue injury management
}

\author{
Carey Middleton McClellan, ${ }^{1}$ Fiona Cramp, ${ }^{2}$ Jane Powell, ${ }^{2}$ \\ Jonathan Richard Benger ${ }^{1,2}$
}

To cite: McClellan CM, Cramp F, Powell J, et al. A randomised trial comparing the cost effectiveness of different emergency department healthcare professionals in soft tissue injury management. BMJ Open 2013;3:e001116. doi:10.1136/bmjopen-2012001116

- Prepublication history and additional material for this paper are available online. To view these files please visit the journal online (http://dx.doi.org/10.1136/ bmjopen-2012-001116).

Received 6 March 2012 Revised 23 November 2012 Accepted 23 November 2012

This final article is available for use under the terms of the Creative Commons Attribution Non-Commercial 2.0 Licence; see http://bmjopen.bmj.com

${ }^{1}$ Academic Department of Emergency Care, University Hospitals Bristol NHS Foundation Trust, Bristol, UK ${ }^{2}$ University of the West of England, Bristol, UK

Correspondence to Dr Carey Middleton McClellan; carey.mcclellan@uhbristol. nhs.uk

\section{ABSTRACT}

Objectives: To evaluate the cost effectiveness of soft tissue injury management by emergency nurse practitioners (ENPS) and extended scope physiotherapists (ESPs) compared with the routine care provided by doctors in an emergency department (ED).

Design: Randomised, pragmatic trial of equivalence.

Setting: A single ED in England.

Participants: 372 patients were randomised, 126 to the ESP group, 123 to the ENP group and 123 to the doctor group. Participants were adults (16 years and older) presenting to the ED with a peripheral soft tissue injury eligible for management by any of the three professional groups.

Interventions: Patients were randomised to treatment by an ESP, ENP or routine care provided by doctors (of all grades).

Main outcome measures: Economic costminimisation evaluation from a funder perspective of the National Health Service, England incorporating analysis of the direct, indirect and tangible costs of care in primary and secondary settings.

Results: From a funder perspective in primary and secondary care, ESPs and ENPs are at best equivalent and could not cost less than routine care. Uncertainty in cost arises from ESPs and ENPs incurring greater indirect costs, such as those associated with follow-up appointments and subsequent primary care visits. Comparison from a funder perspective in secondary care, that is, considering those costs incurred in secondary care alone, demonstrates that ENPs are equivalent in cost to routine care, while ESPs are either equivalent or possibly cheaper than routine care.

Conclusions: These results question the notion that training the healthcare workforce to undertake extensions of their role is generally cost effective. While the randomised trial indicated that the three professional groups have equivalent clinical outcomes, this economic analysis suggests that substitution of routine care with a predominantly ESP or ENP workforce could prove more expensive. Further research is required to understand the underlying reasons for this. The trial has been registered with ISRCTN-ISRCTN 70891354.

\section{ARTICLE SUMMARY}

Article focus

- This study reports a randomised, pragmatic trial of equivalence comparing nurse practitioners and extended scope physiotherapists (ESPs) with the routine care provided by doctors managing minor injuries in the emergency department (ED).

Key messages

- For this patient group, substituting routine care by doctors with an ESP or nurse practitioner workforce is likely to achieve equivalent cost at best. There is, however, a risk that such substitution would result in greater costs from the perspective of the National Health Service, the patient and society. It is the indirect costs associated with care that make ESPs and nurse practitioners less economically attractive.

- While our companion paper demonstrates clinical equivalence between the three professional groups, these economic results challenge the notion that training a non-medical workforce to undertake extensions in their roles is likely to prove cost effective.

Strengths and limitations of this study

- The follow-up period was focused on the first 8 weeks after injury, and there may be important indirect costs that were not captured by this study.

- The research was undertaken at a single centre, and is therefore unlikely to be representative of all the UK ED; further multicentre work is required.

- Since the number of practitioners was relatively small, it is not clear to what extent the findings can be generalised to all ESPs and nurse practitioners working in the ED. Confirmation of these findings in additional settings would be valuable.

\section{INTRODUCTION}

Emergency departments (EDs) are currently a main provider of treatment for minor 
injuries, and annual attendances are expected to increase. ${ }^{1}$ Emergency nurse practitioners (ENPs) are senior nurses with additional training to autonomously assess, diagnose and treat patients with selected urgent conditions, particularly minor illness and injury. ENPs are being increasingly employed in minor injuries care, and are considered an important part of future service delivery. ${ }^{1}{ }^{2}$ Recently, extended scope physiotherapists (ESPs) have also been developed to undertake similar roles, including the management of minor injuries, but this opportunity has not been widely adopted or evaluated. ${ }^{34}$

The new roles of ENP and ESP should benefit both patients and staff. ${ }^{5}$ It is therefore important to establish who has the competencies needed to achieve highquality care in a cost effective manner. ${ }^{5-7}$ A companion paper concluded that the clinical outcomes of soft tissue injury treated by ESPs and ENPs in the ED were equivalent to routine care provided by doctors. ${ }^{8}$ As all groups were clinically equivalent it is other factors such as cost, workforce sustainability, service provision and skill mix that become important in determining the future workforce and models of service delivery.

The aim of this research was to investigate the cost effectiveness of ENPs and ESPs in comparison with the routine care provided by doctors when treating soft tissue injuries in an English ED.

\section{METHOD}

This was a randomised, pragmatic trial of equivalence undertaken in an adult ED in England. The aim was to evaluate and compare the cost effectiveness of the treatment of soft tissue injury by three groups of emergency care professionals: ENPs, ESPs and doctors (of all grades). The full methodology of the randomised clinical trial is detailed in a companion paper. ${ }^{8}$ The economic evaluation, a cost-minimisation analysis, was undertaken from a funder perspective capturing the direct, indirect and intangible costs in primary and secondary care associated with patient care episodes. It was originally intended to undertake a cost utility and consequence analysis, but the economic evaluation took the form of a cost minimisation analysis because the clinical trial demonstrated equivalence of patient outcome between the different healthcare professional groups. The economic information was captured in the first 8 weeks following injury.

The primary economic outcome measures were: the cost per hour of patient contact and the cost per patient per hour. The cost per patient per hour was calculated by dividing the salary cost of the different professional groups by their productivity, that is, the number of patients treated per hour. This provided information on cost in relation to the different productivity levels of different groups. The secondary outcomes were: the direct cost per hour of patient contact and the indirect costs of care per hour of patient contact. All the primary and secondary costs excluded educational costs, as these can be treated as sunk costs or retrospective costs that have been incurred and cannot be recovered. The cost of training all medical professionals is substantial because the level of knowledge and skill required for ED roles requires acquisition of high-quality, costly education and practice. The costs including education have been evaluated as part of a sensitivity analysis.

\section{Direct costs}

The direct costs were obtained by attaching a questionnaire to the patient's notes with a simple tick box system identifying all resources used. The unit cost of different healthcare professionals and follow-up appointments were calculated using the 2007/2008 unit cost of healthcare from the University of Kent. ${ }^{9}$ The cost of different healthcare professionals included the following: salary (as median point on pay scale), salary 'on costs', overheads, capital overheads, travel, contact times and non-London multiplier. The ENP and ESP pay level was calculated using the hospital-based ENP team manager, band 7 Agenda for Change. The doctor's pay level was calculated at a Foundation Trainee 2 level (as no information was available specifically for a SHO or ST1/2 doctor level), specialist registrar and surgical consultant level. All resource costs and calculation methods are detailed in the trial protocol.

\section{Indirect costs}

Indirect costs were obtained during the follow-up telephone interviews using a questionnaire. The direct and indirect costs included in the economic analysis have been summarised in table 1 .

\section{SENSITIVITY ANALYSIS}

A sensitivity analysis was undertaken including the following elements:

- Educational costs (undergraduate, postgraduate and continuous professional development costs from qualification through to retirement).

Table 1 The direct and indirect costs identified and measured in the economic evaluation

\begin{tabular}{|c|c|}
\hline Direct costs & Indirect costs \\
\hline $\begin{array}{l}\text { Resource use } \\
\text { (crutches, tubigrip, etc) }\end{array}$ & $\begin{array}{l}\text { Unplanned further healthcare } \\
\text { visits (eg, to the ED or patient's } \\
\text { general practitioner) }\end{array}$ \\
\hline $\begin{array}{l}\text { Medications } \\
\text { administered }\end{array}$ & $\begin{array}{l}\text { Cost of travel and parking } \\
\text { associated with the above }\end{array}$ \\
\hline $\begin{array}{l}\text { Forward referral to } \\
\text { orthopaedics }\end{array}$ & $\begin{array}{l}\text { Additional items, such as pain } \\
\text { relief or bandages, purchased } \\
\text { since the first visit to the ED for } \\
\text { the injury }\end{array}$ \\
\hline $\begin{array}{l}\text { Forward referral to } \\
\text { physiotherapy }\end{array}$ & $\begin{array}{l}\text { Additional costs to the individual } \\
\text { and others as a direct result of } \\
\text { the injury }\end{array}$ \\
\hline $\begin{array}{l}\text { ED reviews } \\
\text { Forward referral to GP } \\
\text { Cost of practitioner }\end{array}$ & \\
\hline
\end{tabular}


- Median costs (it is recommended that results are presented in mean not median values, which better represent true costs. ${ }^{10}$ The median results are useful as they indicate the cost to the majority of patients).

- ESP and ENPs compared with different grades of hospital doctor.

- Different costs of treating upper-limb and lower-limb injuries.

\section{DISCOUNTING}

In accordance with accepted recommendations, the costs have been presented in two different ways. ${ }^{11}$ The first uses original undiscounted values from 2007/2008. The second uses discounted values from 2007/2008 to 2011 based on the UK government's social time preference rate of $3.5 \% .^{12}$ Using this rate $£ 1$ : 00 in 2007/2008 is worth $£ 0.92$ in $2011 / 2012$. The undiscounted values are presented in the main text, while the discounted values are presented in table 2 .

\section{PRODUCTIVITY}

Patients were not advised to discount the effects of lost productivity when completing the SF-12v2. To eliminate the possibility of double-counting, the effects of lost

\begin{tabular}{|c|c|c|}
\hline Outcome measure & $\begin{array}{l}\text { Undiscounted } \\
\text { cost (£) } \\
2007 / 2008\end{array}$ & $\begin{array}{l}\text { Discounted } \\
\text { cost }(£) \\
2011 / 2012 \\
\end{array}$ \\
\hline \multicolumn{3}{|l|}{ Primary out comes } \\
\hline The mean cost per & Doctor 80.91 & Doctor 74.44 \\
\hline hour of patient care & ESPs 89.71 & ESPs 82.53 \\
\hline $\begin{array}{l}\text { between different } \\
\text { healthcare } \\
\text { professionals }\end{array}$ & ENPs 109.81 & ENPs 101.03 \\
\hline The mean unit & Doctor 54.93 & Doctor 50.54 \\
\hline cost per patient & ESPs 68.36 & ESPs 62.89 \\
\hline per hour & ENPs 86.47 & ENPs 82.31 \\
\hline \multicolumn{3}{|l|}{ Secondary outcomes } \\
\hline The mean direct & Doctor 60.96 & Doctor 56.08 \\
\hline costs per hour of & ESPs 52.48 & ESPs 48.28 \\
\hline patient contact & ENPs 55.21 & ENPs 50.79 \\
\hline The mean indirect & Doctor 19.60 & Doctor 18.03 \\
\hline costs per hour of & ESPs 39.56 & ESPs 36.40 \\
\hline patient contact & ENPs 52.70 & ENPs 48.48 \\
\hline \multicolumn{3}{|l|}{ Sensitivity analysis } \\
\hline The cost per hour & Doctor 92.26 & Doctor 84.88 \\
\hline of patient contact & ESPs 92.71 & ESPs 85.29 \\
\hline $\begin{array}{l}\text { (including } \\
\text { educational costs) }\end{array}$ & ENPs 110.82 & ENPs 101.95 \\
\hline Cost per patient & Doctor 57.42 & Doctor 52.83 \\
\hline per hour (including & ESPs 69.38 & ESPs 63.82 \\
\hline educational costs) & ENPs 86.75 & ENPs 79.81 \\
\hline Median costs & Doctor 48.18 & Doctor 44.32 \\
\hline \multirow[t]{2}{*}{ (all costs) } & ESPs 35.74 & ESPs 32.88 \\
\hline & ENPs 34.58 & ENPs 31.81 \\
\hline
\end{tabular}

ENP, emergency nurse practitioners; ESP, extended scope physiotherapists. productivity on the utility score were not included in the indirect costs.

\section{OPPORTUNITY AND MARGINAL COSTS}

The unit cost of healthcare used in the calculations accommodated the marginal and opportunity costs. ${ }^{9}$

\section{STATISTICAL METHODS}

The sample size was calculated using equivalence margins rather than probability levels: further details are presented in the companion paper. $^{8}$ The trial was powered to $90 \%$ and the minimum importance difference was $10 \%$ of cost. The results were analysed using an intention to treat and per protocol analysis. Economic evaluation data are invariably positively skewed, and it requires an alternative analysis. ${ }^{13} 14$ The study data were analysed using a non-parametric bootstrapping technique. Bootstrapping makes no assumptions regarding the equality, variance or shape of the distribution, and takes into account skewness. ${ }^{15}$ It derives arithmetic means recommended by some authors $^{10}$ that can be used to compare the groups and generates CIs using a computer-generated programme to repeatedly sample from the observed data which is then analysed. ${ }^{1013} 15$ The bootstrapping was undertaken using STATA V.9 software.

\section{RESULTS}

Three hundred and seventy-two patients provided consent and were randomised. One hundred and twenty-six were randomised to the ESP group, 123 to the ENP group and 123 to the doctor group. Table 2 presents the average undiscounted and discounted results. A CONSORT diagram is provided in figure 1 , and details of follow-up in figure 2. Further information can be found in the companion paper. ${ }^{8}$

\section{Primary outcomes}

Cost per hour of patient care between different healthcare professionals

The average cost per hour of patient contact was $£ 80.91$ (CI 66.5 to 101.6) for doctors, $£ 89.71$ (73.0 to 118.7) for ESPs and £109.81 (83.0 to 142.1) for ENPs. The per protocol analysis provided similar findings. The results demonstrated that both the ESP and ENP group could not be cheaper than routine care; they are at best equivalent, and possibly more expensive.

\section{Unit cost per patient per hour}

The ESP group treated an average of 3 patients per hour, whereas the ENPs treated 3.6 and the doctors 4.4 patients per hour. The average cost per patient per hour was $£ 54.93$ (37.9 to 73.0 ) for doctors, $£ 68.36$ (50.6 to 91.3) for ESPs and $£ 86.47$ (62.2 to 122.5) for ENPs. Again, the results demonstrated that both the ESP and 


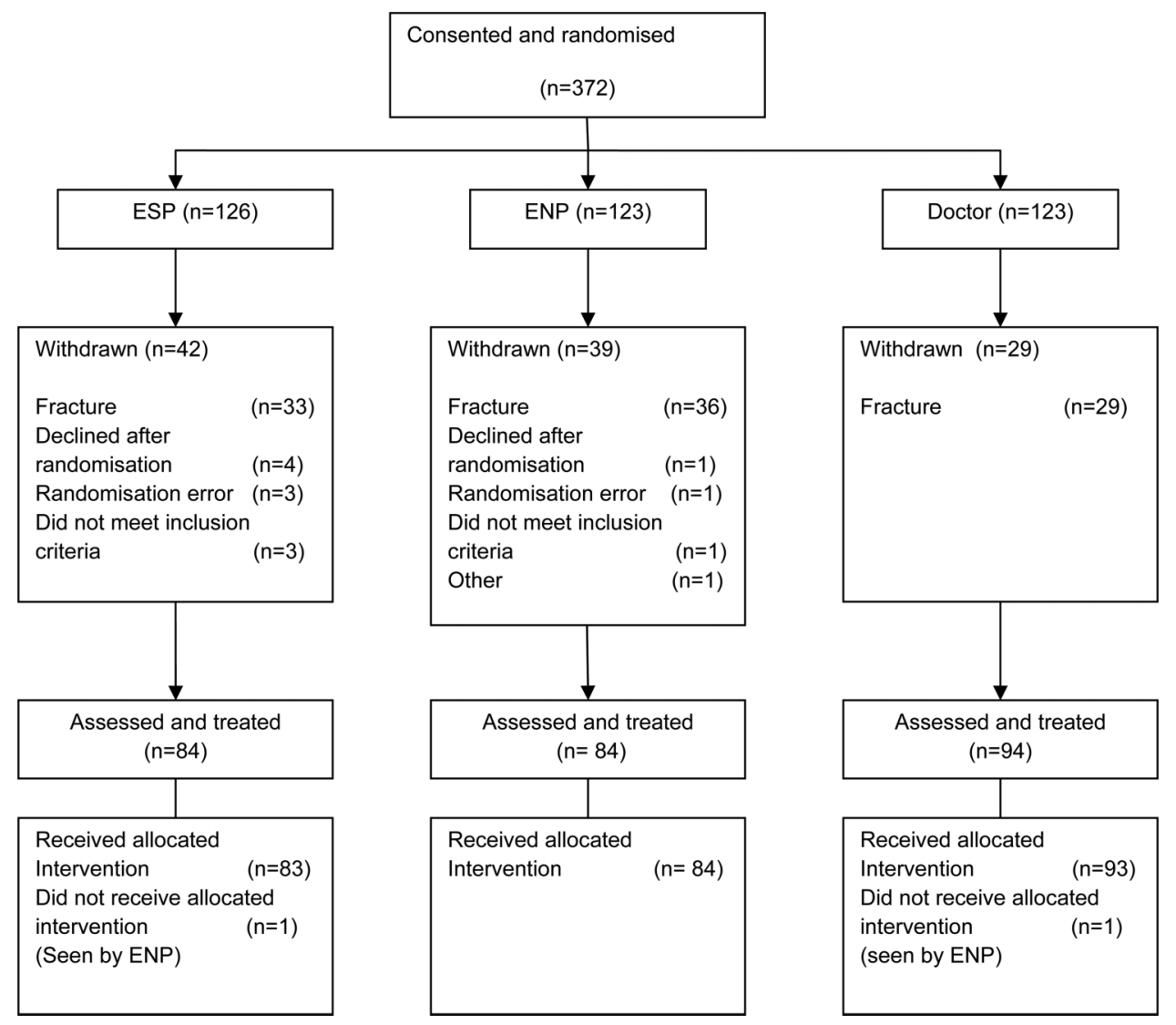

Figure 1 CONSORT statement of trial recruitment.

ENP group could not be cheaper than routine care; they are at best equivalent, and possibly more expensive.

\section{Secondary outcomes: the direct costs (secondary care)}

The average direct cost per hour of patient contact to the National Health Service (NHS) (secondary care) was $£ 60.96$ (51.1 to 73.6) for doctors, £52.48 (44.7 to 63.9) for ESPs and $£ 55.21$ (47.0 to 66.0) for ENPs. The ENP group was equivalent in cost to routine care. The ESP group could not be more expensive than routine care, and was either equivalent or cheaper. Some differences in direct costs between the three groups are noted below:

- In total, $23.5 \%$ of patients were administered medication during their ED visit. The ESP group administered medication to $3.6 \%$ of patients $(n=3)$, the ENPs to $23.2 \%(n=19)$ and the doctors to $42.2 \% \quad(n=38)$ of patients (Pearson's $\chi^{2} \mathrm{p}<0.001$ ).

- The ESP group administered a greater number of crutches $(26.5 \% ; \mathrm{n}=22)$ compared with ENPs and doctors $(9.5 \% ; \mathrm{n}=8$ and $16.5 \% ; \mathrm{n}=15$, respectively).

- The ESP group provided $20.5 \%(\mathrm{n}=17)$ of patients with a broad arm or collar and cuff sling compared with $8.4 \%(n=7)$ in the ENP group and $4.4 \% \quad(n=4)$ in the doctor group.

\section{Secondary outcomes: indirect costs (patient and society)}

The average per patient indirect cost per hour of patient contact associated with the routine care provided by doctors was £19.60 (11.2 to 38.6), compared with £39.56 (25.6 to 59.0) for ESPs and £52.70 (33.0 to 81.6) for ENPs. The ESP and ENP groups could not cost less and were at best equivalent with the doctor group in terms of cost. Important observations regarding the indirect costs were as follows:

- Patients treated by an ENP attended their general practitioner (GP) more frequently $(26.4 \% ; \mathrm{n}=19)$ than those treated by an $\operatorname{ESP}(17.4 \% ; \mathrm{n}=12)$ or a doctor $(13.2 \% ; n=9)$.

- The number of patients referred for an orthopaedic follow-up review was slightly higher in the ENP group $(7.3 \%, \mathrm{n}=6)$, compared with ESPs $(4.8 \%, \mathrm{n}=4)$ or doctors $(5.6 \%, \mathrm{n}=5)$, but the numbers were very small.

- Approximately $60 \%$ of patient who attended the ED with an acute musculoskeletal injury purchased a combination of pain relief, bandages or complementary medicine to assist their recovery. There was no significant difference in purchased items between the groups (Pearsons's $\chi^{2} \mathrm{p}=0.632$ ).

\section{SENSITIVITY ANALYSIS}

Cost per hour of patient contact (including educational costs)

When including educational costs as part of a sensitivity analysis the average costs per hour of patient contact 


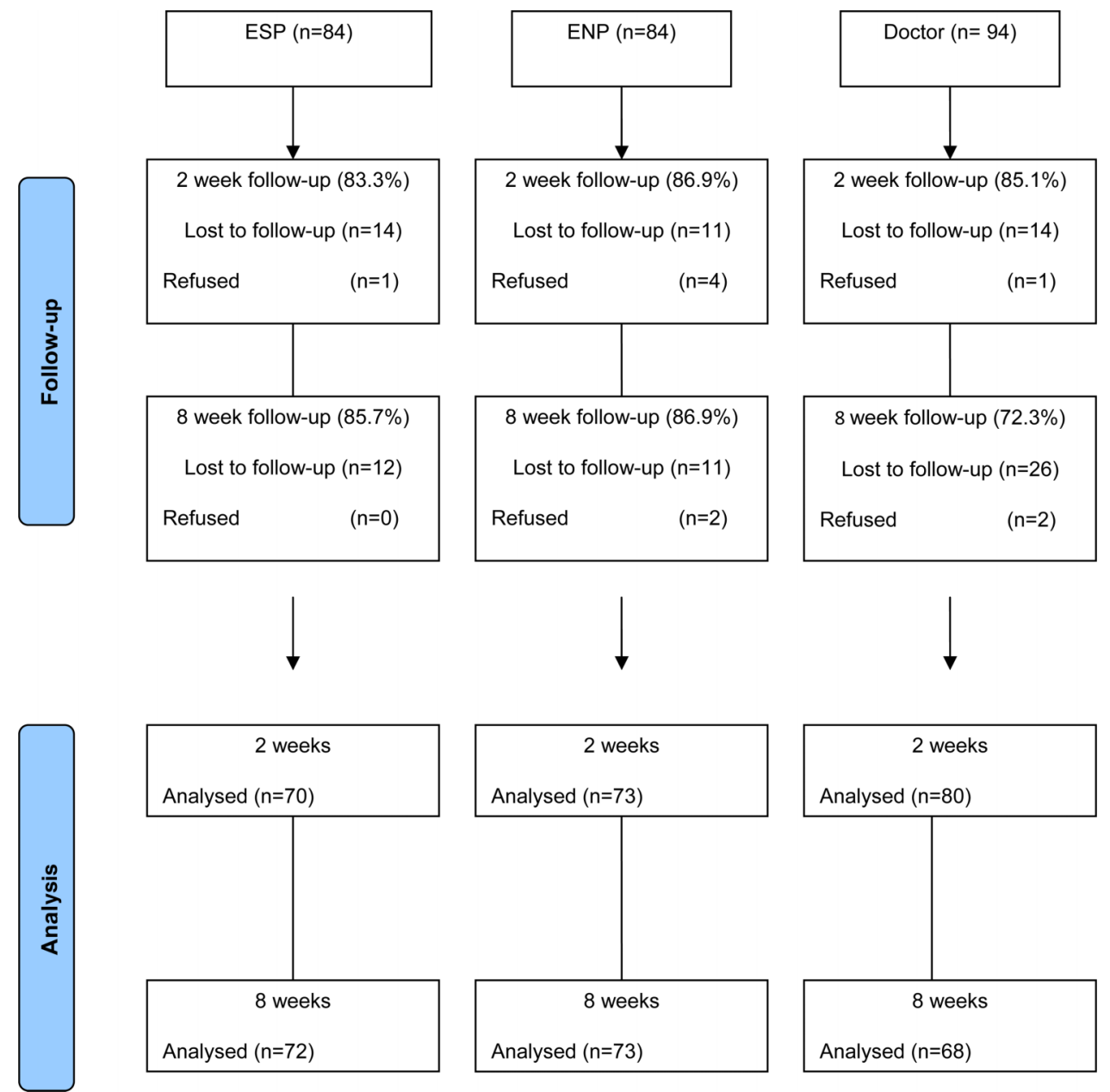

Figure 2 Patient follow-up.

were £92.26 (78.2 to 115.3) for doctors, £92.71 (75.4 to 117.7) for ESPs and £110.82 for ENPs (85.8 to 146.5). In this context the ESP group was equivalent to routine care while the ENP group could not be cheaper, and was either equivalent or possibly more expensive.

\section{Cost per patient per hour (including educational costs)}

When including educational costs the average costs were $£ 57.42$ (43.5 to 81.3) for doctors, £69.38 (51.5 to 96.2) for ESPs and $£ 86.75$ (62.6 to 118.6) for ENPs. This demonstrated that both the ESP and ENP group could not be cheaper than routine care.

\section{Median costs}

The median bootstrap results differed significantly. The average cost per hour was $£ 35.74$ (34.2 to 58.6) for the ESP group, $£ 34.58$ (32.5 to 62.6) for the ENP group and $£ 48.18$ (40.2 to 70.3) for the doctor group. In this analysis the ESP and ENP groups could not be more expensive, and are either equivalent or cheaper. The proportion of cost difference remained the same across all the economic calculations.
ENPs and ESPs compared with junior doctors

ESPs $£ 89.71$ (73.0 to 118.7) were equivalent to junior doctors (Specialist Trainee years 1 and 2: ST1/2) £81.53 (52.4 to 129.7) in all cost calculations. The ENP results $£ 109.51$ (83.0 to 142.1) were more uncertain, but with a trend towards equivalence.

\section{ENPS and ESPs to registrars}

The ESPs £89.71 (73.0 to 118.7) and ENPs £109.51 (83.0 to 142.1) were not equivalent to registrars $£ 73.50$ (54.0 to 104.4) with regard to costs and the results were uncertain, but with a trend towards equivalence. The ESP group, however, was equivalent to registrars when evaluated per hour of patient contact including education costs.

\section{Upper-limb versus lower-limb costs}

Sensitivity analysis demonstrated a consistently higher cost for lower-limb injury compared with upper limb. The average cost for all groups combined, including educational costs, was $£ 81.81$ for upper-limb injury and $£ 98.45$ for lower limb. 


\section{Difference between doctor grades}

A sensitivity analysis was undertaken to evaluate possible differences between junior doctors (ST1/ST2) and more senior grades (ST4-6). A bootstrapping technique indicated that the cost per hour of patient contact was similar: $£ 39.40$ (25.43 to 61.16 ) for ST1/2 doctors and $£ 43.37$ (19.22 to 67.52) for ST4-6 doctors at 8 weeks. However, the unit costs per patient did differ at $£ 30.61$ (10.48 to 46.09 ) for ST1/2 and £11.21 (10.48 to 46.09) for ST4-6.

\section{DISCUSSION}

\section{Statement of principal findings}

We report a randomised trial of equivalence comparing the cost of care by doctors, ENPs and ESPs managing minor injuries in EDs. We have demonstrated that the cost of substituting routine care by doctors with a predominantly ESP or ENP workforce is likely to be at best equivalent in cost with a high likelihood of greater cost from the perspective of the NHS, the patient and society. The cost per hour of patient contact for the different professional groups was $£ 80.91$ for doctors, £89.71 for ESPs and $£ 109.81$ for ENPs. However, an economic analysis undertaken from the perspective of the NHS with costs limited to hospital-based secondary care changes this economic argument. In this context both the ESP and ENP are likely to be equivalent in cost to routine care, and the likelihood of costing less is greater than in the previous comparison. The direct cost per hour of patient contact was $£ 60.96$ for doctors, $£ 52.48$ for ESPs and $£ 55.21$ for ENPs. It is therefore the indirect costs associated with care provided by ESPs and ENPs that make these options for management of soft tissue injuries in ED in England less economically attractive.

\section{Strengths and weaknesses}

This study provides valuable cost-effectiveness data comparing different professional groups which, along with the associated clinical trial, can be used as a basis for decisions relating to workforce and service organisation. Methodological weaknesses have been outlined in the companion paper, ${ }^{8}$ but there are also limitations in this economic. First, the full economic evaluation was only undertaken in the first 8 weeks of injury. It is accepted that there will be additional indirect healthcare costs that have not been captured. It is encouraging that by 8 weeks $86.6 \%$ of patients were 'a lot better' or 'completely better', but there may still be important longer term costs. Second, the accuracy of self-reported time spent with patients by each professional group was not verified. Doctors almost unanimously recorded $10 \mathrm{~min}$ for every patient consultation, whereas the ESPs and ENPs reported a wider spread of contact times. Third, the calculation of opportunity costs needs further consideration. The lack of evidence evaluating the opportunity costs of extended roles has been highlighted by several authors. ${ }^{16-18}$ They have discussed the need to evaluate the consequences of using highly skilled and experienced ENPs and ESPs to undertake new roles. By moving skilled workers away from their existing jobs other consequences follow such as deskilling the existing workforce, the cost associated with any reduction in quality or outcomes of care, the cost of training new staff as a replacement and the impact of the loss of knowledge and clinical skill on a department. It would also be important to consider the consequences of reducing the number of doctors on patient care, future professional skills, postgraduate training and service. The unit cost of healthcare used in these calculations did accommodate the marginal and opportunity costs, ${ }^{9}$ but did not adequately investigate these forgone opportunities. A fourth consideration is that while the Doctor group are likely to be equivalent but possibly cheaper than the ESPs or ENPs the sensitivity analysis showed they were randomised to more of the relatively expensive lowerlimb injuries. This has the potential to overestimate the doctor group's cost. The randomisation process was robust enough to eliminate the possibility of bias and this allocation is likely to be due to chance. A fifth consideration is that at the time this study was conducted it was not routine practice to issue sick notes/fit notes to patients attending the ED, and this applied to all professional groups (including doctors). The practice of issuing sick notes/fit notes to patients varies from hospital to hospital, and may be a factor in subsequent behaviour, but we believe that the type of healthcare practitioner a patient sees may be more influential, and are currently investigating this in further research. Finally, economic evaluation and cost-effectiveness data are useful for comparisons between different services, but there remains some doubt regarding how the information should be applied to inform decision making. ${ }^{19} 20$ Consequently, the findings of economic evaluations are not considered the dominant decisionmaking criterion at a macro, meso or micro level in health services planning. ${ }^{20}$

\section{Comparisons with other studies}

There is only one comparable study: a non-inferiority trial comparing ESPs to routine care provided by ENPs or doctors. ${ }^{21}$ In this trial the researchers estimated that the cost of treatment and indirect costs to patients (using an intention-to-treat analysis) was $£ 92.24$ for a similarly skilled ESP compared with $£ 95.25$ for routine care. These costs are similar to the findings reported here. An association with increased indirect costs for ENPs working in extended roles was similar to previous studies. ${ }^{22}{ }^{23}$ Sakr $e t a l^{22}$ found that ENPs in minor injury units, but not in EDs, had higher follow-up rates which subsequently made the care more expensive than treatment for similar injuries in EDs (by junior doctors). Venning $e t a l^{23}$ found that it was the indirect costs of ENPs in GP practices that added to the overall cost of care. This may indicate a common theme around role 
substitution, and reflects the broader challenges of workforce development within the NHS.

\section{Study implications}

In the current climate of the NHS there are constraints on money, staff and skill mix, time, facilities, equipment and knowledge. ${ }^{11} 13$ While our companion paper demonstrates clinical equivalence between the professional groups, ${ }^{8}$ these economic results challenge the notion that training workforces to undertake extensions in their roles is cost effective. This may have wider implications for the commissioning of services and service delivery, and prove more complicated than a direct workforce substitution. Using economic evidence to make a judgement on present and future service delivery can be difficult, ${ }^{11} 2024-27$ and these results highlight some of the challenges. If the information is used to provide healthcare from the perspective of the NHS, patient and society then ESPs and ENPs are likely to be equivalent in cost, or possibly more expensive. If the service is to be implemented in secondary care alone then the opposite applies. Third, if a view is taken at a national level, planning future service delivery and staffing to include educational costs, then all three groups become more or less equivalent in terms of both clinical quality and cost. At this point it is other factors such as workforce availability and sustainability, service provision and skill mix that become important. The UK College of Emergency Medicine recognises the need to utilise extensions in roles due to increasing patient demand, reducing hours of work for junior doctors and national throughput standards. The use of ENPs and ESPs in extended roles will therefore make a significant contribution to future minor injuries care. ${ }^{28-33}$

\section{Future research}

A further larger scale pragmatic trial of equivalence throughout the UK, with significantly longer follow-up periods and incorporating all the patients managed by the different healthcare professionals, would be valuable but highly challenging. A qualitative study is warranted to explore the subsequent behaviour of patients treated for minor injury in an ED, particularly the factors that determine further unscheduled healthcare contact. Additional research is required to explore the costs and consequences of role substitution, both in EDs and in other areas of the healthcare system.

\section{CONCLUSION}

These results challenge the notion that training the healthcare workforce to undertake extensions in their roles is cost effective. Taken together our two papers have established that ESPs and ENPs have the competencies needed to achieve high-quality clinical outcomes, but they are not necessarily less expensive, depending on the perspective adopted. Further research is required to understand the underlying reasons for this. We have not set out to establish the optimum still mix required, but believe our research will assist in future strategic decision-making.

Contributors CMM initiated the project with input to design and development from FC, JP and JB. CMM collected the data and undertook initial analysis with supervision and additional input from FC, JP and JB. CMM drafted the manuscript which was then revised and approved by FC, JP and JB. CMM acts as guarantor.

Funding The research was funded under a PhD bursary from The University of the West of England.

Competing interests 'All authors have completed the Unified Competing Interest form at http://www.icmje.org/coi_disclosure.pdf (available on request from the corresponding author) and declare that (1) CMM, FC, JP and JB have support from The University of the West of England for the submitted work; (2) CMM, FC,JP and JB have no relationships with The University of the West of England that might have an interest in the submitted work in the previous 3 years; (3) their spouses, partners, or children have no financial relationships that may be relevant to the submitted work; and (4) CMM, FC, $\mathrm{JP}$ and JB have no non-financial interests that may be relevant to the submitted work.'

Ethics approval Ethics approval was obtained by: (1) The Salisbury and South Wiltshire Ethics Committee, obtained on 4 May 2006 (ref: 06/Q2008/10).

(2) The Faculty of Health and Social Care Ethics Committee at the University of the West of England, obtained on 10 July 2006 (ref: HSC/06/07/57).

Provenance and peer review Not commissioned; externally peer reviewed. Data sharing statement There are no additional data are available.

\section{REFERENCES}

1. The College of Emergency Medicine. The way ahead 2008-2012. Statagy and guidance for emergency medicine in the United Kingdom and the Republic of Ireland. London: The College of Emergency Medicine, 2008.

2. The College of Emergency Medicine. Way ahead. London: The College of Emergency Medicine, 2005.

3. Paw RC. Emergency department staffing in England and Wales, April 2007. Emerg Med J 2008;25:420-3.

4. McClellan CM, Greenwood R, Benger JR. Extended scope physiotherapists in the emergency department: what do patients think? Emerg Med J 2006;25:384-7.

5. Department of Health. Freedom to practise: dispelling the myths. London: Department of Health, 2003.

6. Alberti KGMM. Emergency care 10 years on: reforming emergency care. London: Department of Health, 2007.

7. Alberti KGMM. Skillmix: an advance or an excuse? Emerg Med J 2003;20:112-13.

8. McClellan CM, Cramp F, Powell J, et al. A randomised trial comparing the clinical effectiveness of different emergency department healthcare professionals in soft tissue injury management. BMJ Open 2012;2:e001092.

9. Curtis L, Netten A. Unit cost of health and social care. Canterbury: University of Kent, 2007.

10. Thompson SG, Barber JA. How should cost data in pragmatic randomised trials be analysed? BMJ 2000;320:1197-200.

11. Drummond MF, Sculpher M, Torrance GW, et al. Methods for the economic evaluation of healthcare Programs. 3rd edn. New York: Great Britain: Oxford University Press, 2005.

12. HM Treasury. The green book: apprasil and evaluation in central government. London: HM Treasury, 2011.

13. Drummond MF, O'Brien BJ, Stoddart GL, et al. Methods for the economic evaluation of health care programmes. 2nd edn. New York; Oxford: Oxford University Press, 1997.

14. Wiens BL, Iglewicz B. Design and analysis of three treatment equivalence trials. Control Clin Trials 2000;21:127-37.

15. Desgagne A. The use of Bootstrap statistical method for the Pharmaceconomic cost Analysis of Skewed Data. PharmacoEconomics 1998;13:487-97.

16. Tye CC, Ross FM. Blurring boundaries: professional perspectives of the emergency nurse practitioner role in a major accident and emergency department. J Adv Nurs 2000;31:1089-96. 
17. McPherson K. Extended Roles of AHPs in the NHS. A report for the National Co-ordinating Centre for NHS Service Delivery and Organisation R\&D (NCCSDO), 2004.

18. Currie J, Crouch R. How far is too far? Exploring the perceptions of the professions on their current and future roles in emergency care. Emerg Med J 2008;25:335-9.

19. Hoffmann $C$. The influence of economic evaluation studies on decision making. A European survey. Health Policy (serial online) 2000;52:179.

20. van Velden ME. Economic Evaluations of Healthcare Programmes and decision making: the influence of economic evaluations on different healthcare decision-making levels. PharmacoEconomics 2005;23:1075.

21. Richardson B, Shepstone L, Poland F, et al. Randomised controlled trial and cost consequences study comparing initial physiotherapy assessment and management with routine practice for selected patients in an accident and emergency department of an acute hospital. Emerg Med J 2005;22:87-92.

22. Sakr M, Kendall R, Angus J. Emergency nurse practitioners: a three part study in clinical and cost effectiveness. (Research in Sheffield comparing minor injury services provided by nurse practitioners with minor injury care at an A\&E department. 27 refs). Emerg Med $J$ 2003;20:158-63.

23. Venning $\mathrm{P}$, Durie $\mathrm{A}$, Roland $\mathrm{M}$, et al. Randomised controlled tria comparing cost effectiveness of general practitioners and nurse practitioners in primary care. BMJ 2000;320:1048-53.
24. Hoffmann C. The influence of economic evaluation studies on decision making: a European survey. Health Policy 2000;;52:179-92.

25. Jefferson T, Demicheli V, Mugford M. Elementary economic evaluation in healthcare. 2nd edn. London: BMJ Books, 2000.

26. Drummond MF, McGuire A. Economic evaluation of healthcare: merging theory with practice. 1st edn. Oxford: Oxford University Press, 2001.

27. Kelly M, McDaid D, Ludbrook A, et al. Economic apprasil of public health interventions: evidence briefing. London: National Institute for Health and Clinical Excellence, 2005.

28. The NHS plan-A plan for investment. A plan for reform. http://www. dh.gov.uk/en/Publicationsandstatistics/Publications/ PublicationsPolicyAndGuidance/DH_4002960 (accessed 20 Feb 2008).

29. Department of Health. Reforming emergency care, 2001.

30. Department of Health. The Chief Health Professions Officer's ten roles for allield health professionals, 2004.

31. Department of Health. Transforming emergency care in England-a report by Professor Sir George Alberti. England, 2004.

32. Department of Health. Direction of travel: a discussion document London: Department Of Health, 2006.

33. Department of Health. High quality care for all: NHS next stage review final report, 2008. 